\title{
A light scattering study of non equilibrium fluctuations in liquid mixtures to measure the Soret and mass diffusion coefficient
}

\author{
F. Croccolo, ${ }^{1, a)}$ H. Bataller, ${ }^{2}$ and F. Scheffold ${ }^{1}$ \\ ${ }^{1}$ Department of Physics, University of Fribourg, Ch. du Musée 3, CH-1700 Fribourg, Switzerland \\ ${ }^{2}$ Laboratoire des Fluides Complexes et leurs Reservoirs - UMR 5150, Université de Pau et des Pays de \\ l'Adour, BP 1155, 64013 Pau Cedex, France
}

(Received 9 October 2012; accepted 28 November 2012; published online 19 December 2012)

\begin{abstract}
We use dynamic near field scattering to measure the dynamics of concentration non equilibrium fluctuations at the steady-state of Soret separation. The analysis reveals that above a threshold wave vector $q_{c}$, the dynamics is governed by diffusion while at smaller wave vectors, gravity dominates. From the measurements, we extract both the mass diffusion and the Soret coefficients. Comparing our results with literature data, we find good agreement confirming that the proposed experimental technique can be considered a sound approach for the study of thermodiffusion processes. () 2012 American Institute of Physics. [http://dx.doi.org/10.1063/1.4771872]
\end{abstract}

\section{INTRODUCTION}

Non equilibrium fluctuations (NEFs) in fluids can be observed in the presence of a gradient of one (or more) thermodynamic variable. ${ }^{1}$ For example, a temperature gradient in a pure liquid generates unusually strong temperature fluctuations. Equally, a concentration or a temperature gradient in a fluid mixture can provide a huge enhancement in its concentration fluctuations. One interesting aspect of NEFs is that their amplitude increases with size that can lead to mesoscopic and macroscopic fluctuations, which are truly longranged and can be visualized optically. ${ }^{2}$ The amplitude of NEFs as a function of the wave vector $q$ shows a power law dependence $I(q) \propto q^{-4}$, which is limited at small wave vectors due to the influence of gravity and the size of the containing vessel. Theoretically, it was found that fluctuating hydrodynamics (FHD) is well suited (after introducing convenient approximations) for describing both the intensity enhancement and the interplay between diffusion and gravity. ${ }^{3-5}$ Moreover, finite-size effects due to the sample container can be handled by means of more realistic Galerkin approximations. ${ }^{6-9}$ Quite recently, the equations of FHD have also been fully numerically solved in the works of Donev and co-workers ${ }^{10-12}$ providing results, which are in better agreement to experimental microgravity data ${ }^{13}$ than those obtained by means of Galerkin approximations.

From the experimental point of view, different variants of light scattering techniques were used to test theoretical predictions. Both static and dynamic light scattering are sensitive to the Soret and mass diffusion coefficients as well as the critical cut-off wave vector $q_{c}$ set by the influence of gravity. ${ }^{13-20}$ Here, the main experimental challenge is accessing very small wave vectors where the amplitude of NEFs becomes huge. Moreover, investigating very small wave vectors is essential to probe the interplay between the transport processes and gravity occurring at real macroscopic length scales. In 1993, Segrè et $a l .{ }^{3}$ proposed the use of dynamic light scattering

\footnotetext{
${ }^{a)}$ Electronic mail: fabrizio.croccolo@univ-pau.fr.
}

(DLS) to characterize NEFs. Their technique is based on the heterodyne measurement of the temporal correlation function, which can be related to the Soret and mass diffusion coefficient $S_{T}$ and $D$ as outlined below. However, their traditional DLS-approach did not allow probing directly the gravity induced frustration of NEFs at small wave vectors thus limiting the accuracy of their analysis. Following their original work, the values of the Soret coefficient reported by Segrè et al. were found to be inconsistent with data obtained by means of thermal diffusion forced Rayleigh scattering (TDFRS) ${ }^{21}$ and of optical beam deflection (OBD), ${ }^{22}$ with a deviation found to be on the order of $25 \%$.

In this article, we introduce dynamic near field scattering as a powerful tool to study NEFs. Near field scattering (NFS) has been introduced in the late 1990's as an alternative to conventional static and dynamic light scattering. ${ }^{23-26}$ In a NFS experiment, one typically observes the heterodyne contribution of scattered light obtained by interference with the unscattered transmitted beam acting as a local oscillator. Since the primary beam is not masked, the method can provide access to very small scattering angles and corresponding wave vectors. Both a shadowgraph and Schlieren optical layout can be used to implement NFS. ${ }^{15,17,27}$ Here, we show that by using dynamic NFS, we are able to extract both the mass diffusion coefficient $D$ and the critical cut-off wave vector $q_{c}$ from a single experiment. Eventually from those values, we can derive $S_{T}$ and the thermodiffusion coefficient $D_{T}$ as

$$
D_{T}=D \times S_{T} .
$$

Here, we present a comprehensive study of NEFs in three binary liquid mixtures applying and critically testing this approach. $^{28,29}$

As shown later in the text, the Soret and thermodiffusion coefficients derived from our experiments are indeed found in good agreement with literature data. Our results thus confirm the validity of common theoretical concepts on the dynamics of concentration NEFs. The paper is organized as follows: Sec. II describes the experimental setup, Sec. III outlines the 
NFS technique and its implementation, Sec. IV briefly introduces the scattering equations used in Sec. $\mathrm{V}$ for the interpretation of the experimental data. The article closes with a short summary of the main results and concluding remarks in Sec. VI.

\section{THERMODIFFUSION EXPERIMENTS}

Our thermodiffusion cell (see Fig. 1) consists of two square sapphire plates $\left(40 \times 40 \times 8 \mathrm{~mm}^{3}\right)$ kept at different temperatures by two square Peltier elements (Kryotherm, TB-109-1.4-1.5 CH) with a central circular aperture $(\phi$ $=13 \mathrm{~mm}$ ). The two Peltier elements are driven by two temperature controllers (TCs) making use of a proportionalintegral-derivative (PID) feedback system (Wavelength Electronics, TCS651) maintaining the temperature of the internal side of each Peltier element with a stability better than $1 \mathrm{mK}$ RMS over 1 day. Such a high temperature stability is needed in particular when working close to the critical point of the mixture while for the present study a lower accuracy would have been also sufficient. The excess heat at the other side of each Peltier is removed by water circulating in two aluminum plates. The two sapphire windows are kept apart by four adjustable spacers. In our experiments, we set the liquid layer thickness to $h=1.3 \mathrm{~mm}$ or $h=1.53 \mathrm{~mm}$. The sample is horizontally confined within a Viton O-Ring with an internal diameter $d=26 \mathrm{~mm}$.

We perform our experiments by imposing different temperature gradients on the horizontally positioned thin cell containing the initially homogenous fluid mixtures. The samples

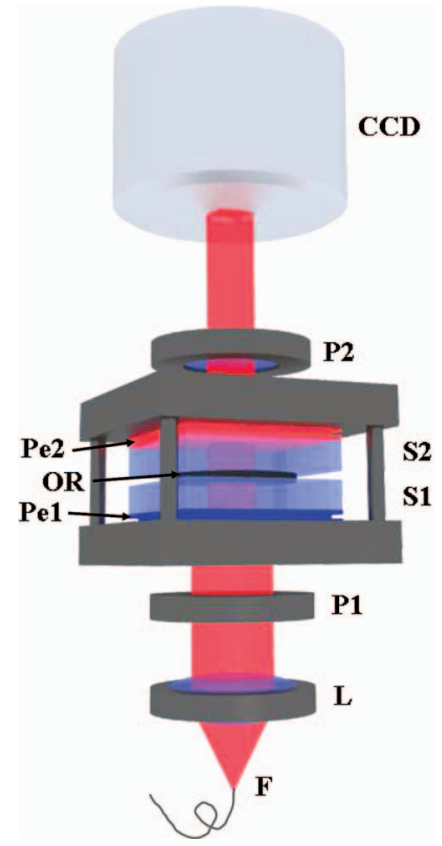

FIG. 1. Experimental setup: a mono-mode optical fiber (F) delivers the low coherent light beam, which is then collimated by a lens (L) and passes a linear polarizer (P1) before entering the thermodiffusion cell. A second linear polarizer (P2) after the sample cell acts as an analyzer to modulate the intensity of light impinging onto the CCD sensor. The liquid mixture is confined horizontally by an O-ring (OR) and vertically by two sapphire plates (S1,S2) in thermal contact with two Peltier elements $(\mathrm{Pe} 1, \mathrm{Pe} 2)$. The excess heat is removed by two water-cooled aluminium plates. are chosen from the Fointainebleu benchmark. ${ }^{30}$ We study three different combinations of $50 \%$ w/w binary mixtures containing the three fluids: 1,2,3,4-tetrahydronaphthalene, isobuthylbenzene, and n-dodecane (in the following, THN, IBB, and n-C12, respectively). The imposed temperature differences span the range from 8 to $20 \mathrm{~K}$. The resulting temperature gradients range from approximately 50 to $150 \mathrm{~K} / \mathrm{cm}$. The mass diffusion coefficients are of the order of $10^{-9} \mathrm{~m}^{2} / \mathrm{s}$ and thus the vertical diffusion time across the layer of thickness $h$ is about $\tau_{d}=h^{2} / D \approx 0.5 \mathrm{~h}$. A typical experimental run consists in applying the temperature gradient to the mixture and letting the Soret separation completely evolve for about $4 \mathrm{~h}$. The chosen cell thickness is $h=1.3 \mathrm{~mm}$ for the THNIBB and the IBB-n-C12 mixtures and $h=1.53 \mathrm{~mm}$ for the THN-n-C12 mixture.

\section{OUTLINE OF DYNAMIC NEAR FIELD SCATTERING EXPERIMENTS}

In a light scattering experiment, monochromatic light with a wave vector $\boldsymbol{k}_{\mathrm{i}}$ impinges on a sample and is scattered in directions $\boldsymbol{k}_{\mathrm{s}}$. For an elastic scattering process $k_{\mathrm{s}}=k_{\mathrm{i}}=k$, the modulus of the momentum transfer is equal to $Q=\left|\boldsymbol{k}_{\mathrm{s}}-\boldsymbol{k}_{\mathrm{i}}\right|=2 k \sin (\theta / 2), \theta$ being the scattering angle. At a point far from the sample, the scattered field $e_{\mathrm{s}}(\boldsymbol{r}, t)$ is a superposition of waves scattered around $\boldsymbol{Q}$. Under near field conditions, $e_{\mathrm{S}}(\boldsymbol{r}, t)$ is composed of contributions arising from many different $\boldsymbol{Q}$. In principle, the individual $\boldsymbol{Q}$ components can be recovered by simply computing the spatial power spectrum of $e_{\mathrm{s}}(\boldsymbol{r}, t),{ }^{25}$

$$
\left|E_{\mathrm{s}}(\boldsymbol{q}, t)\right|^{2}=I_{\mathrm{s}}(\boldsymbol{q}, t),
$$

where $I_{\mathrm{s}}(\boldsymbol{q}, t)$ is the scattered intensity, $E_{\mathrm{s}}(\boldsymbol{q}, t)$ is the spatial Fourier transform of the field distribution $e_{\mathrm{s}}(\boldsymbol{r}, t)$ for $\boldsymbol{r}$ in a plane perpendicular to $\boldsymbol{k}_{\mathrm{i}}$, and $q=Q\left[1-(Q / 2 k)^{2}\right]^{1 / 2}$.

However, practically all scattering experiments are based on measurements of the scattered intensity $i_{\mathrm{s}}(\boldsymbol{r}, t)$ instead of the field amplitude. This complication can be avoided by adopting a heterodyne detection scheme in which the scattered field is mixed with the transmitted one $e_{\mathrm{o}}(\boldsymbol{r})$. For $e_{\mathrm{o}}(\boldsymbol{r}) \gg e_{\mathrm{S}}(\boldsymbol{r}, t)$, the measured intensity is well approximated by $i_{\mathrm{m}}(\boldsymbol{r}, t)=i_{\mathrm{o}}(\boldsymbol{r})+2 R e\left\{e_{\mathrm{s}}(\boldsymbol{r}, t) e_{\mathrm{o}}^{*}(\boldsymbol{r})\right\},{ }^{25}$ where the first term is constant and equal to the intensity of the transmitted beam and the second term is the fluctuating heterodyne signal. By analogy with Eq. (2), $I_{\mathrm{s}}(\boldsymbol{q}, t)$ can be obtained by computing the power spectrum of the heterodyne signal, ${ }^{15}$

$$
\left|I_{\mathrm{m}}(\boldsymbol{q}, t)-I_{\mathrm{o}}(\boldsymbol{q})\right|^{2}=a\left\{T(\boldsymbol{q}) I_{\mathrm{s}}(\boldsymbol{q}, t)+B(\boldsymbol{q})\right\},
$$

where $I_{\mathrm{m}}(\boldsymbol{q}, t)$ and $I_{\mathrm{o}}(\boldsymbol{q})$ are the spatial Fourier transforms of $i_{\mathrm{m}}(\boldsymbol{r}, t)$ and $i_{\mathrm{o}}(\boldsymbol{r}), a$ is a renormalization constant, $T(\boldsymbol{q})$ is the transfer function of the imaging optics, and $B(\boldsymbol{q})$ is the noise of the measurement.

An elegant way to remove the transmitted beam intensity in Eq. (3) is to compute the difference $\Delta i_{\mathrm{m}}(\boldsymbol{r}, t)=i_{\mathrm{m}}\left(\boldsymbol{r}, t^{*}\right)$ $-i_{\mathrm{m}}\left(\boldsymbol{r}, t^{*}+t\right)$ between two measured images separated by a delay time $t .^{15-17}$ For delay times much larger than the relaxation time of the system at a given $\boldsymbol{q}$, the two scattering fields are uncorrelated and the power spectrum $\left|\Delta I_{\mathrm{m}}(\boldsymbol{q}, t)\right|^{2}$ is again proportional to the scattered intensity $I_{\mathrm{s}}(\boldsymbol{q})$. Here, 

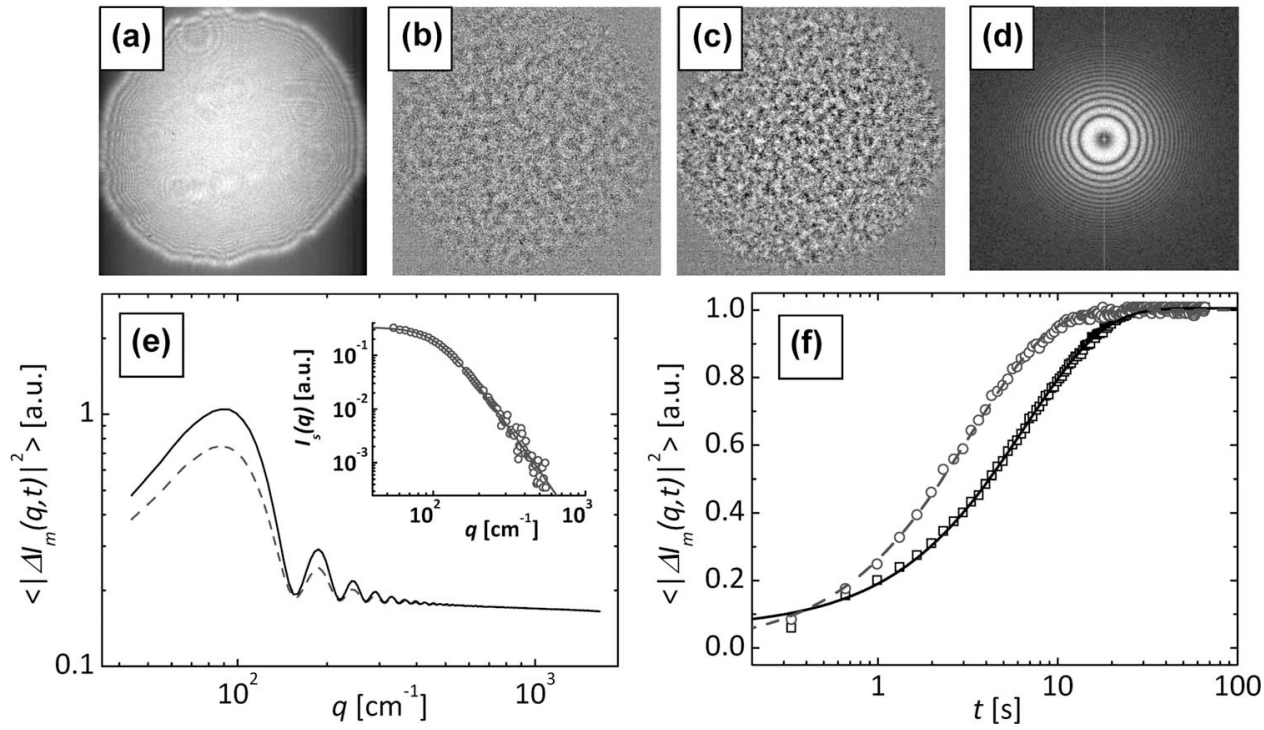

FIG. 2. Results of a near field scattering experiment (Shadowgraph layout) on a binary liquid mixture stressed by a thermal gradient (THN-IBB, $\Delta T=16 K$ ). Upper panels: (a) $2048 \times 2048$ near field image of the sample, (b) and (c) image differences having a time delay of $t=0.33 \mathrm{~s}$ and $33 \mathrm{~s}$, respectively, (d) spatial Fourier transform $\left\langle\left|\Delta I_{\mathrm{m}}(\boldsymbol{q}, t)\right|^{2}\right\rangle$ of (c). Lower panels: Power spectra $\left\langle\left|\Delta I_{\mathrm{m}}(q, t)\right|^{2}\right\rangle$ computed from a set of 3000 images. (e) Results as a function of wave vector $q$ for $t=0.33 \mathrm{~s}$ (dashed gray line) and $33 \mathrm{~s}$ (continuous black line) and (f) as a function of the delay time $t$ for $q=110 \mathrm{~cm}^{-1}$ (black squares) and $q=192 \mathrm{~cm}^{-1}$ (gray circles) together with fitting lines as per Eq. (4), providing time decays of $t=7.1 \mathrm{~s}$ and $3.3 \mathrm{~s}$. Inset: (e) Scattered intensity $I_{\mathrm{s}}(q)$ as obtained after division by the Shadowgraph transfer function and its fit to $I_{0} /\left[1+\left(q / q_{\mathrm{c}}\right)^{4}\right]$, where $\lambda_{c}=2 \pi / q_{\mathrm{c}}=0.6 \mathrm{~mm}$ is the size of the systems critical fluctuations. ${ }^{2}$

$\Delta I_{\mathrm{m}}(\boldsymbol{q}, t)$ is the spatial Fourier transformation of $\Delta i_{\mathrm{m}}(\boldsymbol{r}, t)$. If the system has not relaxed yet, the power spectrum has an additional contribution characteristic of the system's dynamics. More specifically, the temporal evolution of the power spectrum at a given $\boldsymbol{q}$ is given by $[1-f(\boldsymbol{q}, t)]$, where $f(\boldsymbol{q}, t)$ is the normalized intermediate scattering function (ISF), commonly studied in scattering experiments and statistical mechanics,

$$
\left\langle\left|\Delta I_{\mathrm{m}}(\boldsymbol{q}, t)\right|^{2}\right\rangle=2 a\left\{T(\boldsymbol{q}) I_{\mathrm{s}}(\boldsymbol{q})[1-f(\boldsymbol{q}, t)]+B(\boldsymbol{q})\right\},
$$

where $I_{\mathrm{s}}(\boldsymbol{q})=\left\langle I_{\mathrm{s}}(\boldsymbol{q}, t)\right\rangle$ is the ensemble averaged scattered intensity. Provided the dynamics of the system is stationary and the relaxation time scale is short compared to the measurement time, the ensemble average of the power spectrum can be obtained by calculating the time average. For isotropic scattering, additional statistical accuracy is obtained by performing radial averages. In our experiment, we also normalize each image by its spatial average to eliminate the influence of residual fluctuations of the primary beam intensity.

The double-frame differential analysis described above is a generic approach that can be applied in various near field scattering techniques ranging from Shadowgraph and Schlieren detection layouts to near field light and X-ray scattering or differential dynamic microscopy. ${ }^{13,15-17,25,31-38}$ In this work, we have implemented the shadowgraph detection scheme mainly due to the very simple and robust setup and experimental procedures.

In Fig. 1, a sketch of the optical setup is also shown. The shadowgraph makes use of a low coherence light source ( $\mathrm{Su}$ per Lumen Diodes, Broad Lighter S680, $\lambda=680 \pm 10 \mathrm{~nm}$ ) delivered through a single-mode fiber $(\mathrm{F})$. The resulting diverging beam is collimated by an achromatic doublet lens (L, $f=15 \mathrm{~cm}, \phi=5 \mathrm{~cm}$ ) and then passes through a fixed linear film polarizer (P1) in order to set the beam polarization before entering the thermodiffusion cell. A second lin- ear polarizer after the cell (P2) allows us to adapt the average transmitted light intensity. The detection plane is located at about $z=26 \mathrm{~cm}$ from the sample plane. As a sensor, we use a charge coupled device (CCD4000, Vosskühler, Germany) with $2048 \times 2048$ square pixels each of size $7.4 \times 7.4 \mu \mathrm{m}^{2}$ and a dynamic range of 12-bit. After reaching the steady-state of Soret diffusion, sequences of 3000 images are acquired at a frame rate of $3 \mathrm{~Hz}$. Each image contains $2048 \times 2048$ pixels and exposure times are varied from 0.35 to $35 \mathrm{~ms}$ for different runs. After the acquisition, the temperature difference is incremented and a new acquisition run is started after waiting for another $4 \mathrm{~h}$. A resulting near field image is presented in Fig. 2(a). The borders of the cell as well as the Gaussian profile of the primary beam and residual interferences due to reflections and diffraction are visible in the image. However, the subtraction of images efficiently removes these undesired effects as well as stray light contributions yielding a homogeneous speckle pattern $\Delta i_{\mathrm{m}}(\boldsymbol{r}, t)$ similarly to subtracting a reference image. ${ }^{25}$ This is illustrated in Fig. 2 for a time delay of (b) $t=0.33 \mathrm{~s}$, (c) and $33 \mathrm{~s}$. We next compute the time averaged spatial power spectrum of image differences with equal $t$ (Fig. 2(d) for $t=33 \mathrm{~s}$ ). In our experiment, the scattering process is isotropic and the power spectrum can be averaged over scattering vectors having the same modulus $q=|\boldsymbol{q}|$. A selection of power spectra $\left\langle\left|\Delta I_{\mathrm{m}}(q, t)\right|^{2}\right\rangle$ is presented in Figs. 2(e) and 2(f) as a function of $q$ and $t$, respectively. The scattered intensity is obtained by extrapolating the time power spectra towards short and long times. Indeed, the noise contribution $B(q)$ can be extracted from the short time limit of \langle|$\Delta I_{\mathrm{m}}(q$, $\left.t)\left.\right|^{2}\right\rangle$ where $f(q, t \rightarrow 0)=1$, whereas $T(q) I_{\mathrm{s}}(q)$ is obtained at long times where $f(q, t \rightarrow \infty)=0$.

We now turn our attention to the dynamic part of the scattering signal. As mentioned above, the time resolved analysis of the scattering signal provides access to the 
intermediate scattering function of NEFs for each wave vector covered by the NFS experiment and each delay time contained in the image sequence. Working at 1:1 magnification, the theoretically accessible wave vectors are set by the size of the area detector and the pixel size. If $L$ is the edge length of the area detector, then $q$-values range from $q_{\min }=2 \pi / L$ to $q_{\operatorname{Max}}=q_{\min } \times N \sqrt{2} / 2$ where $N^{2}$ is the total number of pixels $(2048 \times 2048$ in our case). In practice, not all the wave vectors can be probed primarily due to a limited sample size and the poor signal to noise ratio at the extremes. This limits the range of accessible wave numbers in our experiment to about $q \in\left[10 q_{\min }, 150 q_{\min }\right]$. The accessible time delays range from $t_{\min }=\Delta t$, set by the interframe time difference, to the maximum value of $t_{\text {Max }}=M \Delta t$ where $M$ is the number of acquired images and therefore in our experiments $t \in[0.33 \mathrm{~s}, 1000 \mathrm{~s}]$.

In addition to the relaxation time, it is possible to extract from our measurements the relative intensity of the scattered signal $I_{s}(q)$. Such a static low angle scattering analysis has already been described in detail elsewhere ${ }^{15,16,39}$ and we will only discuss it here briefly. First, we point out that measured static signal is actually convoluted by the imaging transfer function $T(q)$, which depends on the optical setup. In the case of a shadowgraph layout, the transfer function shows oscillations, which need to be finely characterized before performing a deconvolution..$^{29,39,40}$ The static signals as obtained after division by the Shadowgraph transfer function $I_{S}(q)$, is plotted in the inset of Fig. 2(e) for a typical case. . $^{27,29,39,40}$

Finally, we note that for processing the large amount of data acquired in a single experimental run (about 24 GB), we take the advantage of parallel processing on a graphics processing unit (GPU) with the aim to reduce the computational time to less than one hour per experiment. ${ }^{39}$ Our personal computer motherboard is equipped with an 8-core CPU (INTEL, XEON X3440 at 2.53GHz) and a GPU-board (NVIDIA, Tesla C2050). The double-frame differential analysis is implemented in $\mathrm{C}++/$ CUDA as described in Ref. 39.

\section{THEORY OF LIGHT SCATTERING FROM NON EQUILIBRIUM FLUCTUATIONS}

A binary mixture subject to a thermal stress can undergo mass separation, a phenomenon known as the Ludwig-Soret effect or thermodiffusion. Immediately after applying the thermal gradient, a linear temperature profile is established in the fluid mixture. A dynamic equilibrium is reached once the Soret diffusion is balanced by mass diffusion. The exact time required to reach a stationary state again depends on the mass diffusivity $D$. We note that this picture is valid only for ordinary binary mixtures where the Lewis number $L e=D / D_{T}$ $\ll 1$ where $D_{T}$ is the thermodiffusion coefficient given by Eq. (1). The direction of the concentration gradient is determined by the sign of the Soret coefficient $S_{T}$ defined by an empirical relation valid at steady-state conditions, ${ }^{41,42}$

$$
S_{T}=-\frac{1}{c_{o}\left(1-c_{o}\right)} \frac{\vec{\nabla} c}{\vec{\nabla} T},
$$

where $c_{o}$ is the initial concentration of the denser component of the mixture, $\nabla c$ is the resulting steady-state concentration gradient, and $\nabla T$ is the imposed temperature gradient.
It has been shown by means of fluctuating hydrodynamics that the intensity of the light scattered by concentration NEFs at the steady-state of a Soret-driven separation process under the influence of gravity can be written as 3,5

$$
I_{s}(q)=\frac{I_{o}}{1+\left(q / q_{c}\right)^{4}},
$$

where $I_{o}=\left[k_{B} T /\left(16 \pi^{4} \rho\right)\right] \times\left[\nabla c /(\beta g)-(\partial c / \partial \mu)_{p, T}\right]$ is the intensity of NEFs at the plateau arising due to the quenching effect of gravity for wave vectors $q \ll q_{c}, k_{B}$ is the Boltzmann constant, $T$ is the average temperature of the mixture, $\rho$ is the density, $\beta=1 / \rho(\partial \rho / \partial c)$ is the solutal expansion coefficient, $g$ is the gravitational acceleration, $\mu$ is the chemical potential per unit mass, and $q_{c}$ is the gravitational critical wave vector set by

$$
q_{c}=\left(\frac{\beta \vec{g} \cdot \vec{\nabla} c}{\nu D}\right)^{1 / 4},
$$

where $v$ is the kinematic viscosity of the mixture.

The reported results of FHD are obtained after introducing two main approximations: first, it is assumed that the fluid properties $(v, \rho$, etc. $)$ are constant within the sample; second, periodic or quasi-periodic approximations are involved to make the equations solvable. In the present experimental conditions, the concentration difference in the sample are always smaller than $6 \%$, therefore, we assume that the sample properties are essentially constant within the sample. The latter approximation should not affect the system dynamics, therefore is not relevant to the present study.

At the steady-state of a Soret separation process, the concentration gradient can be related to the temperature gradient via Eq. (5). Thus, the critical wave vector can be expressed in terms of the Soret coefficient

$$
q_{c}=\left[-\frac{\beta \vec{g} \cdot \vec{\nabla} T S_{T} c_{o}\left(1-c_{o}\right)}{\nu D}\right]^{1 / 4} .
$$

The time auto-correlation function $C(q, t)$ probed in dynamic NFS can be expressed as the sum of three different exponentials (for details see, for example, Eqs. (6.1) and (6.2) in Ref. 4). The first term is due to thermal fluctuations, the second to viscous fluctuations, and the third one to concentration fluctuations. In typical binary mixtures, the first two terms decay much faster than the third one and can thus be neglected in our experiments as discussed in Ref. 4. In the following, we will consider only the contribution of concentration NEFs as a single exponential decay $f(q, t)=\exp (-t / \tau(q))$ and thus

$$
C(q, t)=B(q)+I_{s}(q) f(q, t),
$$

where $\mathrm{B}(\mathrm{q})$ is a background noise level, $I_{s}(q)$ is the amplitude of concentration NEFs as described above, and $\tau(q)$ is the time constant at a specific wave vector $q$. The time constant for the concentration component in the presence of gravity can be obtained by combining Eqs. (22) and (25) in Ref. 5 and taking into account the comments made after Eq. (26) in the same article

$$
\tau(q)=\frac{1}{D q^{2}\left[1+\left(q_{c} / q\right)^{4}\right]} .
$$




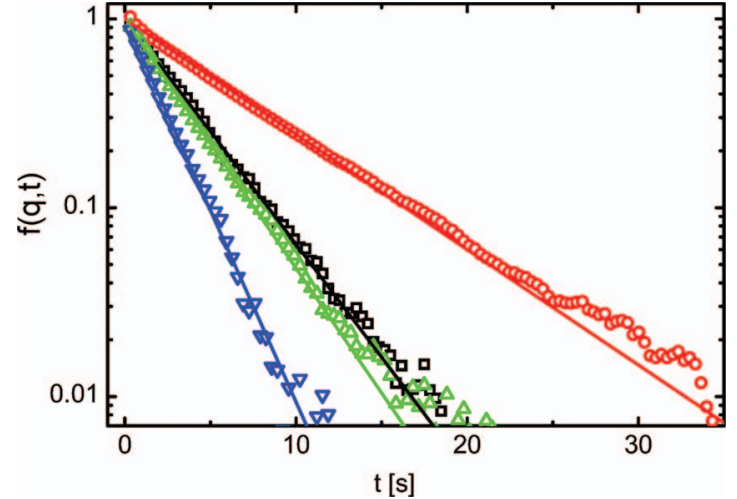

FIG. 3. Normalized intermediate scattering function derived for the sample THN-IBB, stressed by a temperature gradient of $123 \mathrm{~K} / \mathrm{cm}$ for 4 different values of the wave vector $q$, namely: black squares for $q=60 / \mathrm{cm}(\tau=3.6 \mathrm{~s})$, red circles for $q=110 / \mathrm{cm}(\tau=7.1 \mathrm{~s})$, green up-triangles for $q=192 / \mathrm{cm}$ ( $\tau=3.3 \mathrm{~s})$ and blue down-triangles for $q=247 / \mathrm{cm}(\tau=2.1 \mathrm{~s})$.

This result is obtained with the further approximation $q$ $\gg q_{c}(4 D / v)^{1 / 4}$, i.e., in conditions where traveling waves are not present in the system. This approximation fully holds for all our measurements, for example, for the THN-n-C12 sample traveling waves appear for $q<27 \mathrm{~cm}^{-1}$ while our wave vector range starts at $50 \mathrm{~cm}^{-1}$. Here, $q_{c}$ is the same critical value appearing in Eqs. (6)-(8). For wave vectors $q \gg q_{c}$, the fluctuations behave diffusively, while for $q \ll q_{c}$ the effect of gravity (Archimedes' buoyancy) is dominant. ${ }^{2,15-17,43}$ The relaxation time reaches a maximum exactly at $q \equiv q_{c}$.

\section{RESULTS AND DISCUSSION}

By fitting the time dependent part of the experimental power spectra with Eq. (4), we obtain the normalized intermediate scattering functions for every wave vector. In Fig. 3, some typical examples of the intermediate scattering function are shown clearly revealing the monoexponential decay.

The measured relaxation time constants $\tau(q)$ as a function of the wave vector are plotted in Fig. 4 for all three liquid mixtures. As expected, we recover the typical bell-shape predicted for NEFs subject to gravitational forces. As outlined in the literature, ${ }^{5,15-17}$ this shape mirrors the presence of two distinct regimes. For wave vectors larger than the critical value $q_{c}$, the fluctuations are dominated by diffusion, whereas for wave vectors smaller than $q_{c}$, the buoyancy effect of gravity is dominant. The combined action of both mechanisms leads to the $q$-dependence of the relaxation time constant given by Eq. (10). Interestingly, the sensitivity of NEF's to gravity al-

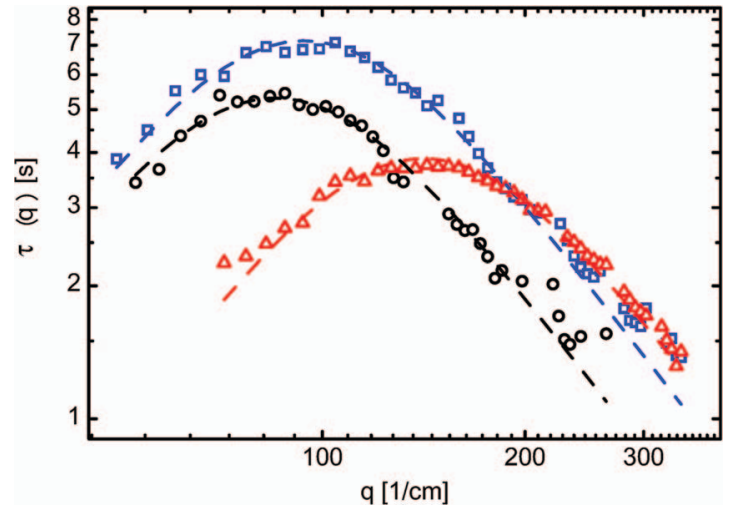

FIG. 4. Time constant of NEFs as a function of the wave vector. Dashed lines show the results of a fit with Eq. (10) for three different samples, namely: red triangles for THN-n-C12 $\left(q_{c}=138.1 / \mathrm{cm}, D=6.6 \times 10^{-10} \mathrm{~cm}^{2} / \mathrm{s}\right)$; blue squares for THN-IBB $\left(q_{c}=93.8 / \mathrm{cm}, D=7.9 \times 10^{-10} \mathrm{~cm}^{2} / \mathrm{s}\right)$; black circles for IBB-n-C12 $\left(q_{c}=88.1 / \mathrm{cm}, D=11.6 \times 10^{-10} \mathrm{~cm}^{2} / \mathrm{s}\right)$. All data shown are for $\Delta T=16 \mathrm{~K}$.

lows us to extract both $q_{c}$ and $D$ independently, provided we can resolve the maximum of $\tau(q)$ with sufficient accuracy as it is the case here (Figure 4). Owing to the high accuracy and the extended $q$-range, we obtain a very robust and accurate fit to the experimental data. Both fitting parameters, $q_{c}$ and $D$, are only weakly correlated. While the former is set by the peak-position, the latter is mostly determined by the power law decay at larger $q$-values. From the measured values of $q_{c}$ and $D$, the Soret coefficient $S_{T}$ can be extracted (Eq. (8))

$$
S_{T}=\frac{q_{c}^{4} \nu D h}{\beta g \Delta T c_{o}\left(1-c_{o}\right)},
$$

taking into account the other known parameters of the fluid mixtures. ${ }^{30}$ From the same data set, the thermodiffusion coefficient can be calculated through Eq. (1).

The experimental values of $D, S_{T}$, and $D_{T}$ are reported in Table I together with literature data ${ }^{30}$ for direct comparison. The reported literature data are obtained by averaging results from different experimental methods, therefore they represent high quality benchmark values for the analyzed mixtures. The values obtained from our measurements using dynamic NFS are in good agreement with literature data. Our results thus indicate that the proposed method is not sensitive to the problems encountered previously in the work of Sengers and coworkers. ${ }^{3}$ We think this is because our experimental approach is based on a coherent analysis of the dynamics fully covering the relevant $q$-range. Sengers and co-workers on the other side, lacking access to wave vectors around $q_{c}$, had to use

TABLE I. Measured and reference values ${ }^{30}$ of the mass diffusion, Soret, and thermodiffusion coefficients of the three analyzed binary mixtures.

\begin{tabular}{|c|c|c|c|c|c|c|}
\hline & \multicolumn{2}{|c|}{$\begin{array}{c}D \\
\left(10^{-10} \mathrm{~m}^{2} \mathrm{~s}^{-1}\right)\end{array}$} & \multicolumn{2}{|c|}{$\begin{array}{c}S_{T} \\
\left(10^{-3} \mathrm{~K}^{-1}\right)\end{array}$} & \multicolumn{2}{|c|}{$\begin{array}{c}D_{T} \\
\left(10^{-12} \mathrm{~m}^{2} \mathrm{~s}^{-1} \mathrm{~K}^{-1}\right)\end{array}$} \\
\hline & This work & Literature $^{30}$ & This work & Literature $^{30}$ & This work & Literature $^{30}$ \\
\hline THN-n-C12 & $6.3 \pm 0.1$ & $6.21 \pm 0.06$ & $9.6 \pm 0.1$ & $9.5 \pm 0.5$ & $6.0 \pm 0.2$ & $5.9 \pm 0.3$ \\
\hline THN-IBB & $7.8 \pm 0.1$ & $8.5 \pm 0.6$ & $3.4 \pm 0.2$ & $3.3 \pm 0.3$ & $2.7 \pm 0.3$ & $2.8 \pm 0.1$ \\
\hline IBB-n-C12 & $10.0 \pm 0.4$ & $9.5 \pm 0.4$ & $3.9 \pm 0.4$ & $3.9 \pm 0.1$ & $3.9 \pm 0.7$ & $3.7 \pm 0.2$ \\
\hline
\end{tabular}


information from static scattering. It remains however unclear whether this discrepancy has its origin in a fundamental discrepancy between (static) scattering theory and experiment or whether it is merely due to limitations in the data analysis procedure implemented in the early work of Sengers and co-workers.

Finally, we wish to emphasize another important advantage of the NFS technique applied to the study of thermodiffusion. In contrast to all the other optical techniques, no prior knowledge about the so-called optical contrast factors $\partial n / \partial T$ and $\partial n / \partial c$ of the mixture is required. This can be an important experimental advantage since these fluid parameters are not always known or accessible, in particular for extreme situations such as high temperatures or high pressure conditions. ${ }^{44,45}$

\section{SHORT SUMMARY AND CONCLUSIONS}

We have performed a near field scattering study of the dynamics of concentration non equilibrium fluctuations during the Soret separation of binary liquid mixtures in a wave vector range well suited for distinguishing the diffusive and gravitational regimes. The analysis of the time constants of NEFs independently provides the mass diffusion and Soret (and thermodiffusion) coefficients of the fluid mixtures under study. Comparing these results with literature data confirms that the proposed experimental technique can be considered a sound approach for the study of thermodiffusion processes.

\section{ACKNOWLEDGMENTS}

F.C. acknowledges financial support from the European Union under Marie Curie Intra European Fellowship (Contract No. IEF-251131), DyNeFI Project. This project has been financially supported by the Swiss National Science Foundation (Project No. 132736) and the Adolphe Merkle Foundation. We thank Frédéric Cardinaux for help in preparing the manuscript.

${ }^{1}$ J. M. Ortiz de Zárate and J. V. Sengers, Hydrodynamic Fluctuations in Fluids and Fluid Mixtures (Elsevier, New York, 2006).

${ }^{2}$ A. Vailati and M. Giglio, "Giant fluctuations in a free diffusion process," Nature (London) 390, 262-265 (1997).

${ }^{3}$ P. N. Segré, R. Schmitz, and J. V. Sengers, "Fluctuations in inhomogeneous and nonequilibrium fluids under the influence of gravity," Physica A 195, 31-52 (1993).

${ }^{4} \mathrm{P}$. N. Segré and J. V. Sengers, "Nonequilibrium fluctuations in liquid mixtures under the influence of gravity," Physica A 198, 46-77 (1993).

${ }^{5}$ A. Vailati and M. Giglio, "Nonequilibrium fluctuations in time-dependent diffusion processes," Phys. Rev. E 58, 4361-4371 (1998).

${ }^{6}$ J. M. Ortiz de Zárate, R. P. Cordón, and J. V. Sengers, "Finite size effects on fluctuations in a fluid out of thermal equilibrium," Physica A 291, 113-130 (2001).

${ }^{7}$ J. M. Ortiz de Zárate and J. V. Sengers, "Boundary effects on the nonequilibrium structure factor of fluids below the Rayleigh-Bénard instability," Phys. Rev. E 66, 036305 (2002).

${ }^{8}$ J. M. Ortiz de Zárate, F. Peluso, and J. V. Sengers, "Nonequilibrium fluctuations in the Rayleigh-Bénard problem for binary fluid mixtures," Eur. Phys. J. E 15, 319-333 (2004).

${ }^{9}$ J. M. Ortiz de Zárate, J. A. Fornés, and J. V. Sengers, "Long-wavelength nonequilibrium concentration fluctuations induced by the Soret effect," Phys. Rev. E 74, 046305 (2006).

${ }^{10}$ A. Donev, J. B. Bell, A. de la Fuente, and A. L. Garcia, "Diffusive transport by thermal velocity fluctuations," Phys. Rev. Lett. 106, 204501 (2011).
${ }^{11}$ A. Donev, J. B. Bell, A. de la Fuente, and A. L. Garcia, "Enhancement of diffusive transport by non-equilibrium thermal fluctuations," J. Stat. Mech. 2011, P06014.

${ }^{12}$ F. Balboa Usabiaga, J. B. Bell, R. Delgado-Buscalioni, A. Donev, T. G. Fai, B. E. Griffith, and C. S. Peskin, "Staggered schemes for fluctuating hydrodynamics," Multiscale Model. Simul. 10, 1369-1408 (2012).

${ }^{13}$ A. Vailati, R. Cerbino, S. Mazzoni, C. J. Takacs, D. S. Cannell, and M. Giglio, "Fractal fronts of diffusion in microgravity," Nat. Commun. 2, 290 (2011).

${ }^{14} \mathrm{~A}$. Vailati and M. Giglio, "q divergence of nonequilibrium fluctuations and its gravity induced frustration in a temperature stressed liquid mixture," Phys. Rev. Lett. 77, 1484 (1996).

${ }^{15}$ F. Croccolo, D. Brogioli, A. Vailati, M. Giglio, and D. S. Cannell, "Use of dynamic schlieren to study fluctuations during free diffusion," Appl. Opt. 45, 2166-2173 (2006).

${ }^{16}$ F. Croccolo, D. Brogioli, A. Vailati, M. Giglio, and D. S. Cannell, "Effect of gravity on the dynamics of non equilibrium fluctuations in a free diffusion experiment," Ann. N.Y. Acad. Sci. 1077, 365-379 (2006).

${ }^{17}$ F. Croccolo, D. Brogioli, A. Vailati, M. Giglio, and D. S. Cannell, "Nondiffusive decay of gradient driven fluctuations in a free-diffusion process," Phys. Rev. E 76, 41112 (2007).

${ }^{18}$ A. Oprisan, S. Oprisan, and A. Teklu, "Experimental study of nonequilibrium fluctuations during free diffusion in nanocolloids using microscopy techniques," Appl. Opt. 49, 86-98 (2010).

${ }^{19}$ A. Oprisan and A. L. Payne, "Dynamic shadowgraph experiments and image processing techniques for investigating non-equilibrium fluctuations during free diffusion in nanocolloids," Opt. Commun. 290, 100-106 (2012)

${ }^{20}$ C. J. Takacs, A. Vailati, R. Cerbino, S. Mazzoni, M. Giglio, and D. S. Cannell, "Thermal fluctuations in a layer of liquid $\mathrm{CS}_{2}$ subjected to temperature gradients with and without the influence of gravity," Phys. Rev. Lett. 106, 244502 (2011).

${ }^{21}$ W. Köhler and B. Müller, "Soret and mass diffusion coefficients of toluene/n-hexane mixtures," J. Chem. Phys. 103, 4367-4370 (1995).

${ }^{22}$ K. J. Zhang, M. E. Briggs, R. W. Gammon, and J. V. Sengers, "Optical measurement of the Soret coefficient and the diffusion coefficient of liquid mixtures," J. Chem. Phys. 104, 6881-6892 (1996).

${ }^{23}$ M. Giglio, M. Carpineti, and A. Vailati, "Space intensity correlations in the near field of the scattered light: A direct measurement of the density correlation function g(r)," Phys. Rev. Lett. 85, 1416-1419 (2000).

${ }^{24}$ D. Brogioli, A. Vailati, and M. Giglio, "Heterodyne near-field scattering," Appl. Phys. Lett. 81, 4109 (2002).

${ }^{25}$ F. Ferri, D. Magatti, D. Pescini, M. A. C. Potenza, and M. Giglio, "Heterodyne near-field scattering: A technique for complex fluids," Phys. Rev. E 70, 041405 (2004).

${ }^{26}$ R. Cerbino and A. Vailati, "Near-field scattering techniques: Novel instrumentation and results from time and spatially resolved investigations of soft matter systems," Curr. Opin. Colloid Interface Sci. 14, 416-425 (2009).

${ }^{27}$ F. Croccolo and D. Brogioli, "Quantitative Fourier analysis of schlieren masks: The transition from shadowgraph to schlieren," Appl. Opt. 50, 3419-3427 (2011)

${ }^{28}$ The present work substantially expands a preliminary study (Ref. 29) on a single binary mixture from the Fointanebleu benchmark (Ref. 30).

${ }^{29} \mathrm{~F}$. Croccolo, F. Scheffold, and H. Bataller, "Mass transport properties of the tetrahydronaphthalene/n-dodecane mixture measured by investigating non equilibrium fluctuations," C. R. Mec. (in press).

${ }^{30}$ J. K. Platten, M. M. Bou-Ali, P. Costesèque, J. F. Dutrieux, W. Köhler, C. Leppla, S. Wiegand, and G. Wittko, "Benchmark values for the Soret, thermal diffusion and diffusion coefficients of three binary organic liquid mixtures," Philos. Mag. 83, 1965-1971 (2003).

${ }^{31}$ R. Cerbino and V. Trappe, "Differential dynamic microscopy: Probing wave vector dependent dynamics with a microscope," Phys. Rev. Lett. 100, 188102 (2008).

${ }^{32}$ R. Cerbino, L. Peverini, M. A. C. Potenza, A. Robert, P. Bösecke, and M. Giglio, "X-ray-scattering information obtained from near-field speckle," Nat. Phys. 4, 238-243 (2008).

${ }^{33}$ L. G. Wilson, V. A. Martinez, J. Schwarz-Linek, J. Tailleur, G. Bryant, P. N. Pusey, and W. C. K. Poon, "Differential dynamic microscopy of bacteria mobility," Phys. Rev. Lett. 106, 018101 (2011).

${ }^{34}$ M. Reufer, V. A. Martinez, P. Schurtenberger, and W. C. K. Poon, "Differential dynamic microscopy for anisotropic colloidal dynamics," Langmuir 28, 4618-4624 (2012).

${ }^{35}$ V. A. Martinez, R. Besseling, O. A. Croze, J. Tailleur, M. Reufer, J. Schwarz-Linek, L. G. Wilson, M. A. Bees, and W. C. K. Poon, 
"Differential dynamic microscopy: A high-throughput method for characterizing the motility of microorganisms," Biophys. J. 103, 1637-1647 (2012).

${ }^{36}$ D. Magatti, M. D. Alaimo, M. A. C. Potenza, and F. Ferri, "Dynamic heterodyne near field scattering," Appl. Phys. Lett. 92, 241101 (2008).

${ }^{37}$ D. Brogioli, D. Salerno, F. Croccolo, R. Ziano, and F. Mantegazza, "Speckles generated by skewed, short-coherence light beams," New J. Phys. 13, 123007 (2011).

${ }^{38}$ D. Salerno, D. Brogioli, F. Croccolo, R. Ziano, and F. Mantegazza, "Photon correlation spectroscopy with incoherent light," Opt. Express 19, 2641626422 (2011).

${ }^{39}$ G. Cerchiari, F. Croccolo, F. Cardinaux, and F. Scheffold, "Note: Quasireal-time analysis of dynamic near field scattering data using a graphics processing unit,” Rev. Sci. Instrum. 83, 106101 (2012).
${ }^{40}$ S. P. Trainoff and D. S. Cannell, "Physical optics treatment of the shadowgraph," Phys. Fluids 14, 1340 (2002).

${ }^{41}$ W. Köhler and S. Wiegand, Thermal Non-Equilibrium Phenomena in Fluid Mixtures (Springer, Berlin, 2002).

${ }^{42}$ S. de Groot and P. Mazur, Non-Equilibrium Thermodynamics (Dover, New York, 1984).

${ }^{43}$ D. A. Weitz, "Diffusion in a different direction," Nature (London) 390, 233-235 (1997).

${ }^{44}$ F. Croccolo, M. A. Arnaud, D. Bégué, and H. Bataller, "Concentration dependent refractive index of a binary mixture at high pressure," J. Chem. Phys. 135, 034901 (2011).

${ }^{45}$ F. Croccolo, F. Plantier, G. Galliero, G. Pijaudier-Cabot, M. Z. Saghir, F. Dubois, S. Van Vaerenbergh, F. Montel, and H. Bataller, "Note: Temperature derivative of the refractive index of a binary mixtures by using a new thermodiffusion cell," Rev. Sci. Instrum. 82, 126105 (2011). 